Foro Interno. Anuario de Teoría Política

ISSN: 1578-4576

http://dx.doi.org/10.5209/FOIN.57204

\title{
Big Data, saber-poder y pastoreo digital: sobre el fundamento mitológico de la autoridad
}

Javier Cigüela Sola ${ }^{1}$

Recibido: 17 de marzo de 2017 / Aceptado: 24 de mayo de 2017

Resumen: Partiendo del intenso impacto que está teniendo el big data y el resto de tecnologías conexas en nuestra existencia personal y social, el presente artículo busca, en primer lugar, realizar una aproximación a la naturaleza del conocimiento que los datos masivos generan desde el concepto foucaultiano de saber-poder, como también desentrañar el modo en que dicho conocimiento es utilizado para legitimar decisiones que se toman en la incertidumbre. En segundo lugar, intentará analizar qué novedad supone dicho saber en relación a su más inmediato precedente: la estadística y, por último, tendrá como fin examinar el tipo de poder que surge de dicho saber, en relación especialmente con las ideas, de nuevo en la estela de Michel Foucault (1926-1984), acerca del poder pastoral y la conducción a través de la verdad confesada.

Palabras clave: Big Data; saber/poder; legitimación; estadística; poder pastoral; Foucault.

\section{[en] Big Data, Knowledge-power and Digital Pastoral Practices: the Mytho- logical Foundations of Authority}

\begin{abstract}
On the basis of the intense impact that Big Data and related technologies are having on our personal and social existence, this article aims first of all to unravel - using the Foucauldian concept of knowledge/power - the nature of the knowledge provided by mass data collection as well as the way in which mining massive data sets are a means to legitimize decisions taken in uncertainty. Secondly, the novelty of this knowledge in relation to its most immediate precedent, statistics, will be analyzed. Finally, the type of power that emerges from this specific knowledge will be examined, especially in connection to ideas, also from Michel Foucault (1926-1984), of pastoral power and conduction through
\end{abstract} confession.

Keywords: Big Data; knowledge-power; legitimation; statistics; pastoral power; Foucault.

Cómo citar: Javier Cigüela Sola, "Big Data, saber-poder y pastoreo digital: sobre el fundamento mitológico de la autoridad”: Foro Interno. Anuario de Teoría Política, vol. 17 (2017), pp. 35-54.

\section{Introducción}

Constituye ya un lugar común la afirmación de que el big data y el resto de tecnologías conexas están transformando no solo nuestra existencia como personas individuales, sino también - y acaso especialmente - nuestra existencia social y política,

\footnotetext{
1 Universitat Abat Oliba / Universitat Oberta de Catalunya

E-mail: jciguelas@uao.es / jciguela@uoc.edu
} 
como ciudadanos de una urbe cuyas decisiones políticas están tomadas cada vez más sobre la base de procesos tecnológicamente condicionados ${ }^{2}$. Fue Marshall McLuhan (1911-1980) quien mejor describió de qué modo la tecnología predominante en cada era transforma tanto al hombre mismo como al entorno que habita: "Todos los medios, desde el alfabeto fonético al ordenador, son extensiones del hombre que transforman su entorno y que le provocan cambios profundos y duraderos"; la tecnología, afirma, es "la extensión de nuestros propios cuerpos"3. La imprenta transformó de modo radical la sociedad y fue el germen de la modernidad, otorgando una formidable importancia al medio escrito y condicionando tanto el surgimiento del Estado moderno burocrático como también la propia revolución industrial. La electrónica hizo lo propio en el siglo veinte, acelerando los procesos de comunicación e interacción y haciendo del mundo una aldea global ${ }^{4}$. En la actualidad, un proceso análogo está en marcha con internet y el conjunto complejo de tecnologías a él conectadas, desde el big data hasta el Internet de las cosas, cuyas consecuencias en nuestra vida personal y social solo estamos comenzando a atisbar ${ }^{5}$.

Sin embargo, al igual que sucedió anteriormente con otros saberes, el big data está influyendo en nuestro hábitat social y personal de un modo que excede sus propios propósitos funcionales: ya no se trata solo de un conjunto de herramientas con las que analizamos más datos y más rápido, sino que está produciendo efectos muy significativos en nuestra vida personal, social y política, en el modo que habitamos nuestro propio cuerpo (piénsese en las técnicas de recogida de datos sobre nuestra salud) y nuestras propias ciudades (piénsese en la Smart Mobility). Nuestra vida personal está cada vez más presionada por el interés de diferentes poderes en recoger datos sobre nosotros. Nuestra vida social lo está también de modo creciente por los datos y algoritmos que influyen en nuestras interacciones; del mismo modo que nuestra vida política está crecientemente conectada a decisiones cuya base empírica o legitimante es precisamente el análisis de datos masivos. Desde la información que obtenemos de otros en las redes sociales hasta el negocio crediticio, pasando por los

2 Así, aunque no se trate de un libro académico (o precisamente por ello y por el recibimiento mediático que ha tenido), ver: Yuval Yoah Harari, Homo Deus. Breve historia del mañana, Debate, Barcelona, 2016, pp. 400 y ss., quien eleva el big data no solo a la categoría de revolución científica sino además a la de creencia religiosa y motor de la vida diaria.

3 Marshall McLuhan, "La entrevista de Playboy", en Carlos Scolari (ed.), Ecología de los medios. Entornos, evoluciones e interpretaciones, Gedisa, Barcelona, 2015, pp. 50 y ss.

4 McLuhan, Comprender los medios de comunicación. Las extensiones del ser humano, Paidós, Barcelona, 1996. A McLuhan le interesaba mucho más hacer antropología que política de los medios, pero lo cierto es que también la forma de gobernarnos mutuamente está mediáticamente condicionada. En realidad, tanto la era disciplinaria como la biopolítica a las que se refiere constantemente Foucault fueron productos directos de la mirada sobre el mundo que Johannes Gutenberg (circa 1400-1468) inauguró con la imprenta: la mirada panóptica se basa justamente en una preponderancia del sentido óptico que no existió hasta que se instauró el texto (leído visualmente) como forma principal de comunicación, allá por el siglo dieciséis, en detrimento de otras formas de percibir y comunicar como la oralidad, central en la tradición medieval, o el olfato, crucial para el hombre cazador-recolector. La imprenta va "de la mano" de la industrialización disciplinaria, sostiene McLuhan, porque el modo lineal, repetitivo, secuencial y mecánico de pensar la producción en masa no es sino una concreción de esos mismos modos textuales de pensar. Lo mismo se puede decir de la biopolítica: "Con la imprenta — dice McLuhan-, fueron posibles la homogeneidad del dinero, los mercados y el transporte, creando por tanto unidades políticas y económicas" que serían entonces los propios Estados centralizados; el proceso estadístico, los registros y la burocracia que están detrás de la vigilancia biopolítica tampoco serían posibles sin una "velocidad en el movimiento de la información impensable antes de la imprenta". McLuhan, "La entrevista de Playboy", pp. 51 y ss.

$5 \quad$ Byung-Chul Han, La sociedad de la transparencia, Herder, Barcelona, 2013, p. 11. 
diagnósticos sanitarios y las investigaciones criminales, todo está siendo transformado por este nuevo conocimiento ${ }^{6}$.

Como señalan Boyd y Crowford, lo anterior hace del big data un fenómeno que no solo concierne a lo tecnológico y lo analítico - sus funciones más obvias-, sino también a lo mitológico, esto es: al modo en que construimos la verdad sobre nosotros mismos y sobre nuestras sociedades, al modo en que asociamos a dicho fenómeno determinadas visiones tanto utópicas como distópicas, y al modo en que lo concebimos como la solución a problemas inmemoriales a la vez que como el origen de nuevas amenazas ${ }^{7}$. El presente artículo tiene como objeto desentrañar el tipo de significaciones que surgen cuando el big data y el resto de tecnologías exceden sus propios propósitos y pasan a formar parte esencial de la construcción de un régimen de verdad y de nuevas formas de circulación del poder.

\section{Big data, el saber-poder y la paradoja de la decisión}

El big data ha revolucionado las relaciones entre el saber y el poder, y lo ha hecho de diferentes maneras. La más evidente es que pone a disposición de quien pueda acceder a ella una cantidad masiva de información que puede ser utilizada para tomar decisiones con amplias consecuencias sociales y no de carácter netamente político. Que "el saber es poder" es algo más que un dicho popular. La relación entre ambos fenómenos ha sido ampliamente tematizada en la filosofía contemporánea, especialmente en la obra de Michel Foucault. Para el pensador francés, la separación conceptual entre saber y poder esconde siempre el hecho de que en la realidad social uno y otro están siempre intensamente imbricados: "Con Platón se inicia el gran mito occidental: lo que de antinómico tiene la relación entre el poder y el saber". Según dice el mito, "si se posee el saber es preciso renunciar al poder; allí donde están el saber y la ciencia en su pura verdad jamás puede haber poder político". Según Foucault, "hay que acabar con este gran mito...por detrás de todo saber o conocimiento lo que está en juego es una lucha por el poder. El poder político no está ausente del saber, por el contrario, está tramado con este"s.

Son muchas las formas en las que el poder y el saber se entrelazan entre sí, formas que van modificándose a lo largo del tiempo y que en cada era conforman un determinado "régimen del saber". Foucault lo expresó de modo muy claro en una entrevista:

Lo importante, creo, es que la verdad no está fuera del poder, ni sin poder...La verdad es de este mundo; está producida aquí gracias a múltiples imposiciones. Tiene aquí efectos reglamentados de poder. Cada sociedad tiene su régimen de verdad, su "política general de la verdad": es decir, los tipos de discursos que ella acoge y hace funcionar como verdaderos; los mecanismos y las instancias que per-

6 Nicholas Carr, Superficiales. ¿Qué está haciendo internet con nuestras mentes?, Taurus, Madrid, 2011, pp. 17 y ss.; Lev Manovich, "Trending: The Promises and the Challenges of Big Social Data” (2011), p. 1. Disponible en: http://manovich.net/content/04-projects/067-trending-the-promises-and-the-challenges-of-big-socialdata/64-article-2011.pdf (11-1-17).

7 Danah Boyd y Kate Crawford, "Critical Questions for Big Data: Provocations for a Cultural, Technological, and Scholarly Phenomenon": Information. Communication \& Society, vol. 15, n. ${ }^{\circ} 5$ (2012), p. 663.

8 Michel Foucault, La verdad y las formas jurídicas, Gedisa, Barcelona, 2008, pp. 60 y ss. 
miten distinguir los enunciados verdaderos o falsos, la manera de sancionar unos y otros; las técnicas y los procedimientos que son valorizados para la obtención de la verdad; el estatuto de aquellos encargados de decir qué es lo que funciona como verdadero'.

En toda era, por tanto, es posible adivinar en dicho entramado - el que acoge los poderes y los saberes y los hace funcionar - una política de la verdad, esto es, una estrategia en la que el propio saber encuentra su lugar y queda políticamente validado, o en la que, por el contrario, queda ocultado o deslegitimado. Así, los diferentes poderes que han existido históricamente han legitimado su modo de funcionar recurriendo muy a menudo a diversos saberes: el poder punitivo que un día se apoyó en el saber religioso y moral, a partir del siglo diecinueve comenzó a legitimarse a través del saber científico-social, y concretamente de la psiquiatría ${ }^{10}$. El poder político que un día confió su legitimación a los saberes filosóficos y teológicos, a partir del siglo diecisiete y dieciocho la encontró en la economía y la estadística, centros de saber políticamente útiles que aún hoy siguen teniendo un peso legitimador importante ${ }^{11}$. Por otro lado, todos estos saberes han sido producidos no en situaciones abstractas sino en relaciones concretas de poder. El psiquiatra que en el siglo diecinueve y aún hoy trata al delincuente para identificar el origen de su desviación, lo hace desde una posición de poder frente al interrogado cuya confesión pretende ${ }^{12}$. Del mismo modo que el Estado que elabora estadísticas y censos de población, que interroga a los enfermos y que los categoriza lo hace desde una posición de soberanía frente a ellos.

Pues bien, en la era digital las relaciones entre saber y poder se están transformando, y una de las causas de dicha transformación reside en la creciente importancia del big data en ámbitos socio-políticos relevantes. Por un lado, hace ya tiempo que se incorporó a los procesos de decisión política como conocimiento útil, en funciones y aspectos sociales tan variados como la gestión del transporte, la práctica sanitaria, la investigación criminal, la economía colaborativa o las campañas electorales. En todos estos campos la gestión y las decisiones se vienen realizando sobre la base del conocimiento masivo y actualizado que proporcionan sobre sí mismos los usuarios, datos que actualmente van desde su ubicación hasta sus gustos y preferencias, pasando por sus estilos de vida e incluso sus hábitos más íntimos. Se habla de modo creciente de Smart Cities, Smart Governance o Smart Management ${ }^{13}$, siendo el Smart

9 “The important thing here, I believe, is that truth isn't outside power, or lacking in power:...truth isn't the reward of free spirits, the child of protracted solitude, nor the privilege of those who have succeeded in liberating themselves. Truth is a thing of this world: it is produced only by virtue of multiple forms of constraint. And it includes regular effects of power". "Each society has its regime of truth, its 'general politics' of truth — that is, the types of discourse it accepts and makes function as true: that is, the types of discourse which it accepts and makes function as true; the mechanisms and instances which enable one to distinguish true and false statements, the means by which each is sanctioned; the techniques and procedures accorded value in the acquisition of truth; the status of those who are charged with saying what counts as true". Michel Foucault, Power/Knowledge. Selected Interviews \& Other Writings 1972-1977, The Harvester Press/Vintage Books, New York, 1980, p. 131.

10 Michel Foucault, Los anormales, Akal, Madrid, 2001.

11 Michel Foucault, Seguridad, Territorio, Población, Fondo de Cultura Económica, Buenos Aires, pp. 293 y ss.

12 Michel Foucault, Wrong-Doing, Truth-Telling. The Function of Avowal in Justice, University of Chicago Press, Chicago, London, 2014.

13 Helmut Willke, Smart Governance: Governing the Global Knowledge Society, Campus Verlag, New York, 2007. 
de todas esas construcciones conceptuales acerca del poder, en buena parte, un calificativo referido al uso de las herramientas de análisis de datos.

Una de las consecuencias tradicionales del entrelazamiento entre el poder y el saber es que este es instrumentalizado por aquel como medio de legitimación de sus decisiones: "Ordeno, entre otras cosas, por lo mucho que sé", diría el poder. En lo que respecta al big data, independientemente de que este influya o mejore las decisiones tomadas por quien ostenta el poder en una determinada situación, su utilización se esgrime como modo de legitimación de la decisión misma, como productor de seguridades cognitivas frente a un entorno de progresiva incertidumbre. Lo anterior cobra especial importancia en las circunstancias del mundo contemporáneo. Así, la creciente complejidad social, técnica y económica está conduciendo a los tradicionales decision makers (operadores políticos y jurídicos, básicamente) a lo que Niklas Luhmann (1927-1998) describe como la "paradoja de la decisión": el juez, el político y el legislador están obligados a decidir, pero lo están en ámbitos respecto a los cuales no tienen prácticamente certezas, sectores que cambian aceleradamente, que requieren un conocimiento técnico especializado y que están afectados por factores incontrolables ${ }^{14}$. El mundo interconectado del que hablaba ya McLuhan produce complejidad por encima de lo que el sistema político o jurídico pueden abarcar, lo que colisiona con el principio de que toda decisión política o jurídica es a la vez inaplazable: "La decisión justa —escribe Jacques Derrida (1930-2004) — es necesaria siempre inmediatamente, enseguida, lo más rápido posible. La decisión no puede procurarse una información infinita y un saber sin límite acerca de las condiciones, las reglas o los imperativos hipotéticos que podrían justificarla" ${ }^{15}$.

Ante la necesidad de decidir en la incertidumbre, al sistema y a sus decisores solo les queda legitimar su decisión por la vía del argumento del conocimiento ex ante: "A lo mejor no supe o pude anticipar las consecuencias de mi decisión —dirá el poder-, pero como mínimo acumulé toda la información posible para tomarla, y además yo era el único que tenía acceso a ella". Es ahí donde el big data entra en juego como conocimiento que reduce la (sensación de) complejidad. Puede ser que no aporte esa información infinita que sería necesaria, pero el dogma que circula entre los dataístas es que los datos masivos de los que hoy disponemos se acercan bastante a un conocimiento infinito y actualizado, y si analizamos las cosas desde un punto de vista puramente cuantitativo lo cierto es que no les faltaría razón. El big data consigue, por tanto, reducir la paradoja de la decisión a su mínimo exponente - "con tanto conocimiento es difícil equivocarse"-, y desde luego genera la expectativa de eliminarla por completo - “con la información adecuada, pronto será imposible equivocarse"- ${ }^{16}$.

Evidentemente, nada de lo anterior garantiza el éxito de la decisión, es posible que incluso un exceso de información sea negativo a la hora de decidir ${ }^{17}$. Pero eso poco importa: en relación a las técnicas de legitimación (y el conocimiento es una de ellas) lo relevante no es el éxito de la decisión misma sino la producción de un

14 Niklas Luhmann, Organización y decisión, Herder/Universidad Iberoamericana, Mexico D.F., 2010; ver también, en lo que respecta al derecho: Niklas Luhmann, Das Recht der Gesellschaft, Suhrkamp, Frankfurt, 1995, pp. 309 y ss.

15 Jaques Derrida, Fuerza de Ley. El "fundamento místico de la autoridad”, Tecnos, Madrid, 2010, p. 61.

16 Nuval Yoah Harari, Homo Deus. Breve historia del mañana, Debate, Barcelona, 2016, pp. 400 y ss.

17 Boyd y Crawford, "Critical Questions for Big Data", p. 668. 
estado de opinión positivo y tranquilizador en torno a ella. Es importante hacer creer al afectado que la decisión se toma en base a certezas científicas, más allá de la probabilidad de éxito. La información es importante, sostiene Luhmann, para generar "ilusiones de control" sobre las consecuencias de una decisión que es en buena medida arbitraria ${ }^{18}$.

Es ahí, por tanto, donde radica el carácter mitológico de esta tecnología: el big data forma parte actualmente del proceso de mistificación propio de toda autoridad que decide autorreferencialmente ${ }^{19}$. Por ello su acceso es necesariamente limitado, y por ello el activismo del open data encuentra tantas resistencias. La razón por la que los gobiernos y las corporaciones como Apple, Facebook o Twitter limitan el acceso a sus datos no es solo que estos tengan una utilidad competitiva y estratégica ${ }^{20}$, sino también y especialmente que dichos datos forman parte de lo que Derrida identifica con el fundamento místico de su autoridad. El big data, como también los algoritmos que regulan la sociedad digital, son lo "indescifrable" y lo "ininterpretable" del poder $^{21}$, el arcano que le da superioridad sobre los afectados. Estos, por su parte, solo pueden esperar a que eso que es ininteligible se manifieste como poder transformador de la realidad, como poder benéfico, como se verá más adelante.

Por último, del mismo modo que ha sucedido tradicionalmente con los saberes políticamente útiles, también el big data produce un saber extraído en determinadas relaciones de poder. De hecho, estas relaciones son, en la era digital, extremadamente asimétricas: la parte que proporciona el conocimiento (el ciudadano observado, el consumidor analizado) tiene una posición muy débil frente a la otra que lo extrae, lo utiliza y lo explota (la corporación o el Gobierno), en la medida en que las condiciones de la relación son innegociables en la mayor parte de casos, y en tanto que uno no puede llevar adelante la actividad, sea la compra de un billete online o el uso de una aplicación en el móvil, sin proporcionar antes los datos exigidos. La relación es tan asimétrica, sostiene Harcourt, que no requiere siquiera de una coerción o una disciplina, ni siquiera se puede hablar propiamente de extracción: muy al contrario, "la estamos proporcionando libre y voluntariamente, con amor, deseo y pasión - a veces, con ambivalencia y duda"-, en cada acto de nuestra vida cotidiana en la red, cada vez que hablamos por Skype con nuestras parejas, que guardamos un archivo en Dropbox o que renovamos una subscripción a una revista digital ${ }^{22}$. Todas estas actividades "aparentemente gratis" estimulan de modo permanente nuestro deseo, y todas ellas llevan implícita una transferencia de información personal exhaustiva que tiene un enorme valor de mercado y que resulta para quien la recibe prácticamente gratis. Los datos que a un Estado de la era estadística le costaba años de elaboración e ingentes recursos, hoy los consiguen Facebook o Instagram con una mera actualización de sus servidores.

"Poder y saber son", en fin, fenómenos "correspondientes, correlativos, superpuestos" "23, y partiendo de que el big data no constituye ninguna excepción a dicha regla, lo cierto es que el saber-poder que funda está tanto o más restringido y

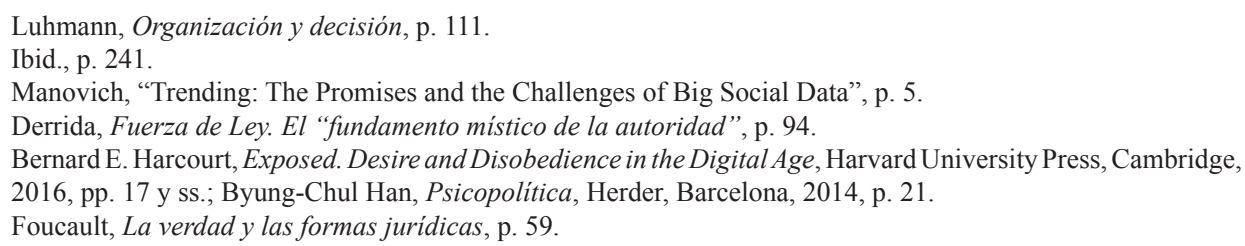


desigualmente distribuido como lo estuvieron los anteriores. A continuación analizaré brevemente la relación entre este nuevo saber y su más inmediato precedente, el saber estadístico, para concluir con el tipo de poder al que aquel está principalmente conectado.

\title{
3. Big data: ¿continuación y/o ruptura del saber estadístico?
}

El precedente histórico más claro de lo que actualmente constituye el big data fue la estadística: según el crítico Byung-Chul Han, "la actual euforia con el Big Data se asemeja a la euforia con la estadística del siglo XVIII"24. La estadística fue, afirma, el motor de la primera ilustración, en la que se creía que iba a ser posible "liberar el conocimiento del contenido mitológico", y derribar prejuicios fundados tan solo en la falta de conocimiento científico. El big data, por su parte, vendría a constituir una segunda ilustración, atravesada por el imperativo de la transparencia ${ }^{25}$, y en la que late la creencia de que la enorme cantidad de información accesible y analizable va a hacer de la propia teoría algo superfluo. El fin de la teoría, incluso de la ciencia, lo predica el archicitado - y un tanto exagerado — artículo de Chris Anderson en la revista Wired:

\begin{abstract}
Ahora hay una mejor manera. Los petabytes nos permiten decir: "La correlación es suficiente". Podemos dejar de buscar modelos. Podemos analizar los datos sin hipótesis sobre lo que podrían mostrar. Podemos lanzar los números a los equipos de computación más grandes que el mundo haya visto y dejar que los algoritmos estadísticos encuentren patrones donde la ciencia no es capaz ${ }^{26}$.
\end{abstract}

Lo cierto es que nada de lo anterior es nuevo, pues el fin de las teorías especulativas o filosóficas ha sido anunciado con cada gran avance científico a lo largo de la historia moderna. No solo la estadística en el siglo dieciocho, ya antes la geometría o las matemáticas fueron anunciadas como los auténticos saberes que iban a reemplazar a los grandes modelos teóricos. Eso mismo escribió Gottfried Leibniz (1646-1716) en 1666 respecto de las matemáticas aplicadas al cálculo, tras la lectura de la obra de Ramon Lull (1232-1316): "Según esto — escribió- cuando surja una controversia, no habrá ya más necesidad de discusión entre dos filósofos de la que hay entre dos calculadores. Bastará con coger la pluma, sentarse y decirse el uno al otro: ¡calculemos!"'27.

Más allá de esta discusión, lo cierto es que el imperativo actual de la transparencia del que habla Han está produciendo importantes efectos, concretamente en lo que a la datificación de nuestro mundo respecta. Los países, las economías, las ciudades,

\footnotetext{
24 Han, Psicopolítica, p. 87.

25 Han, La sociedad de la transparencia, p. 11.

26 "There is now a better way. Petabytes allow us to say: 'Correlation is enough'. We can stop looking for models. We can analyze the data without hypotheses about what it might show. We can throw the numbers into the biggest computing clusters the world has ever seen and let statistical algorithms find patterns where science cannot". Chris Anderson, "The End of Theory: The Data Deluge Makes the Scientific Method Obsolete": Wired (23-6-2008). Disponible en: https://www.wired.com/2008/06/pb-theory/ (11-1-2017).

27 Gottfried W. Leibniz, Leibniz: Selections, ed. y trad. de P. P. Wiener, Scribner, New York, 1951, p. 51.
} 
las dinámicas de los consumidores y usuarios, el transporte, la ciencia, el turismo, en fin, todo está siendo traducido a "flujos de datos"; hasta las propias personas están convirtiéndose en quantified selfs, y todos nuestros movimientos, nuestros gustos y preferencias, nuestras amistades y nuestros estados de ánimo son detalladamente registrados por los dispositivos tecnológicos que llevamos acoplados al cuerpo ${ }^{28}$. El conocimiento que está puesto hoy a disposición de cualquiera de las empresas que gestionan nuestra información, sea Apple, Google o Facebook, es inmensamente mayor que el que gestionaba cualquier monarca soberano del siglo diecinueve, y en ese sentido la segunda ilustración está produciendo un régimen o infraestructura del saber cualitativamente distinto a sus precedentes.

Frente a ello podría argumentarse que el conocimiento producido en la era del big data implica tan solo diferencias cuantitativas frente al que se producía en la era estadística, en el sentido de que el único cambio es que hoy en día accedemos a más datos que antes. Sin embargo, actualmente la compilación y tratamiento de la información es demasiado diferente como para situar la diferencia en el matiz. La primera distancia entre la estadística y el big data tiene que ver con que cuando la diferencia cuantitativa es demasiado grande entonces estamos ya ante algo cualitativamente distinto. Del mismo modo que la diferencia cuantitativa entre tener cien euros y cien millones de euros en la cuenta distingue cualitativamente al pobre del rico, la diferencia entre los pocos millones de datos que existían en 1950 y los cientos de billones de gigabytes de datos que existen hoy supone, en efecto, que estamos frente a realidades distintas. Como se desprende del título de uno de los conocidos artículos de la revista especializada Wired: "More isn't just more - more is different" (Más no es solo más; más es diferente) $)^{29}$.

La segunda diferencia radicaría en el hecho de que la estadística, a pesar de su enorme importancia en todas las ramas de las ciencias sociales, constituía un conocimiento cuyo ámbito de influencia fue más o menos restringido. Es cierto que modificó el modo en que se legitimaba el Estado (de hecho, el término statistik viene de Staat, Estado, y significó en su origen "ciencia del Estado"), como también modificó el curso de diversas ciencias como la economía o la ciencia política, pero no fue más allá de ello. El big data, por el contrario, tiene un efecto expansivo muy superior, es mucho más ubicuo ${ }^{30}$. No solo las ciencias sociales están mutando por la cantidad de datos que existen acerca de las dinámicas políticas, jurídicas o económicas que ellas estudian, sino que todo el resto de ciencias (biología, medicina, física, etc.), como también las profesiones y la propia economía están transformándose por las tecnologías de análisis de datos. La misma vida cotidiana está mutando por el imperio del dato, en la medida en que muchas de las actividades en que nos vemos inmersos tienen que ver, cuando no es directamente su finalidad principal, con la extracción de datos personales.

La tercera diferencia, relacionada con la anterior, es que el big data ha venido acompañado, y sin duda entrelazado, con otras "tecnologías" como el hardware, el software o el internet de las cosas, las cuales están multiplicando sus efectos y condicionando el modo en el que los propios datos son registrados, analizados y uti-

28 Harcourt, Exposed; Benjamin Bratton, The Stack. On Software and Sovereignty, MIT Press, Cambridge, London, 2015.

29 Marian Bantjes, “The Petabyte Age: Because More Isn’t Just More -More Is Different”: Wired (23-6-2008). Disponible en: https://www.wired.com/2008/06/pb-intro/ (11-1-2017).

30 David Bollier (Rapporteur), The Promise and Peril of Big Data, Aspen Institute, Washington, 2010, p. 1. 
lizados para tomar decisiones. Pongamos por ejemplo la relación entre los datos y la tecnología del software: gracias al carácter dinámico del software actual, lo que antes eran documentos o archivos estáticos ahora se convierten en ejecuciones dinámicas de información ${ }^{31}$. Así, si el investigador policial del siglo veinte tenía que buscar a un sospechoso con la última foto y la última localización de que dispusiera en su pila de archivos, muy probablemente antiguas y obsoletas, el investigador actual muy fácilmente tendrá fotografías, ubicaciones y todo lo imaginable acerca del individuo en cuestión del último mes, probablemente del último día, incluso de la última hora o el mismo instante en que lo busca. Es más, gracias a las técnicas de predicción de la tecnología actual, que incorporan las correlaciones, el investigador podrá incluso anticipar sus movimientos futuros ${ }^{32}$. Pero además de dinámica y predictiva, la tecnología del software actual es interactiva, lo que modifica totalmente el modo en que se genera el conocimiento sobre nosotros, nuestras identidades y nuestras relaciones La elaboración estadística del siglo diecinueve requería de poca participación por parte del usuario (acaso una respuesta en un cuestionario anual, o una visita al registro cuando se compra la casa), mientras que hoy en día nuestra implicación en la recogida de datos es absoluta y permanente, está implícita en cada clic que hacemos en nuestros ordenadores, en cada transacción y en cada servicio que contratamos. Es una participación, además, en buena medida inconsciente ${ }^{33}$, lo que hace que la información sea más verosímil. A diferencia de lo que respondemos en las tradicionales encuestas, los datos que proporcionamos en internet y las redes sociales (gustos, preferencias, intereses) reflejan mucho mejor nuestra personalidad en la medida en que no tenemos la sensación de estar siendo interrogados cuando los transmitimos.

Como consecuencia, los datos que se generan son infinitamente mayores, pero también más actualizados, lo que nos lleva a una ulterior diferencia: el proceso de recogida, análisis y procesamiento de datos es tan acelerado que su incorporación como conocimiento disponible no solo se produce a la hora de decidir en tiempo real, sino también para predecir dinámicas o procesos futuros. Así, mientras que la creación de estadísticas requería de una elaboración humana y por ello era dilatada en el tiempo (en series anuales, muy típicamente), los datos actuales son recogidos y procesados de modo prácticamente instantáneo, precisamente porque, en la era de la ciencia post-humana ${ }^{34}$, no son ya predominantemente humanos quienes tratan los datos, sino máquinas inteligentes y algoritmos complejos. Así, el operario de una institución que desea regular el tráfico de una Smart City no solo dispone a golpe de clic de la situación del tráfico en ese mismo momento, sino que, en virtud de la información acumulada y procesada, puede prever los movimientos subsiguientes

31 Lev Manovich, El software toma el mando, UOC Press, Barcelona, 2013, pp. 57 y ss. En su análisis —muy influido por McLuhan - del modo en que el software condiciona los procesos socio-culturales, Manovich destaca que es su carácter dinámico e interactivo lo que produce efectos que contrastan con los de tecnologías anteriores: el archivo digital que utiliza por ejemplo la policía actualmente es mucho más dinámico que el antiguo archivo de papel, en la medida en que los registros se van actualizando automáticamente con transferencias de información que recibe de otros medios y registros; al mismo tiempo, es mucho más interactivo, pues dicha información no es solo una extracción unidireccional que ha hecho la policía mediante sus investigaciones, sino que se alimenta de todo un conjunto de informaciones que circulan por la red y que en muchas ocasiones ha sido proporcionada por el propio usuario investigado. El medio - en este caso la nueva tecnología del software-, modifica la práctica misma a la que se aplica.

32 Galit Shmueli, “To Explain or to Predict?”: Statistical Science, vol. 25, n. 3 (2010), pp. 289-310.

33 Manovich, "Trending: The Promises and the Challenges of Big Social Data”, p. 5.

34 Rosi Braidotti, Lo Posthumano, Gedisa, Barcelona, 2015, pp. 171 y ss. 
y actuar conforme a tal previsión, en un sentido que en otra era resultaba del todo inimaginable ${ }^{35}$.

Hay, por último, otra diferencia relevante que afecta al big data como tecnología del saber, y es su suma complejidad. En el centro de dicha complejidad están los conocidos algoritmos: fórmulas altamente complejas cuyo contenido decide cosas tan relevantes como qué aparece asociado en los buscadores a nuestro nombre, y en qué orden, y que funcionan como auténticas cajas negras ${ }^{36}$. Volviendo a la idea de Derrida, lo cierto es que estos algoritmos indescifrables —al menos en aparienciaconstituyen en buena medida el "fundamento místico de la autoridad" de quienes los controlan. De estas cajas negras solo sabemos qué introducimos (nuestra búsqueda) y qué sale de ellas (los resultados), sin que podamos alcanzar un conocimiento siquiera aproximado de su funcionamiento interno. La complejidad es tal que buena parte del activismo digital aboga, por un lado, por una mayor transparencia en lo que a su contenido respecta $\mathrm{y}$, por otro, por una mayor alfabetización digital que permita empoderar al usuario frente al uso de unas fórmulas que, a la postre, van a determinar importantes aspectos de su vida. Aquí de nuevo lo relevante no es la complejidad real del algoritmo en sí, sino el mito que emerge del conocimiento que genera, la sorpresa del usuario medio al comprobar que las ofertas que recibe on line están adaptadas a sus preferencias, o la confianza del empresario medio en que es el algoritmo digital y no otra tecnología lo que va a proporcionarle el secreto de la búsqueda de clientes.

La relación entra la estadística y el big data estaría determinada, en resumen, por tres notas características: en primer lugar, se puede hablar de cierta evolución de la primera a la segunda, en la medida en que ya desde 1970 y 80 la estadística se había vuelto más compleja y capaz de llevar a cabo grandes acumulaciones de datos a raíz de su contacto con la informática. En segundo lugar, se puede hablar sin duda de coexistencia y complementariedad, pues la estadística sigue siendo crucial, a pesar de la llegada del big data, como régimen de saber-poder en ámbitos variados como las políticas públicas o el urbanismo; pero también se pueden constatar, por último, algunas diferencias cualitativas, en las que me he centrado con mayor detenimiento, en la medida en que el conocimiento que el big data produce no solo es cuantitativamente muy superior sino que además es más dinámico y envuelve de modo más intenso al propio usuario, que participa de modo inconsciente en la elaboración del propio saber que sobre él se produce.

\section{Big data y poder pastoral en la era digital}

Las relaciones entre saber y poder son complejas y bidireccionales. Que el saber sea utilizado para mistificar el ejercicio del poder no excluye en absoluto su capacidad para transformar los efectos que produce. No hay duda, por ejemplo, de que la psiquiatría no solo ha tenido un papel importante en lo que a la construcción de un discurso sobre la locura respecta, pues también lo ha tenido respecto a los propios

35 Alessandro Mantelero, "Smart cities, movilidad inteligente y protección de los datos personales": IDP. Revista de Internet, Derecho y Politica, n. ${ }^{\circ} 21$ (2015), pp. 37-49.

36 Frank Pasquale, The Black Box Society. The Secret Algorithms that Control Money and Information, Harvard University Press, Cambridge, 2015. 
locos, en la medida en que las técnicas de control y exclusión que se les han aplicado a lo largo de la modernidad, desde el electroshock hasta la farmacología, han sido recomendadas o validadas por el propio discurso científico.

En ese sentido, la irrupción de la tecnología digital está produciendo modos completamente nuevos de circulación del poder. Para explicarlos se han tratado de actualizar metáforas recurrentes a lo largo de la modernidad - destacadamente la del panóptico, pero también la del Gran Hermano orwelliano o la de Matrix-, al tiempo que se han tratado de ensayar otras nuevas - Harcourt habla, por ejemplo, de un "pabellón de espejos" en el que aparecemos transparentes a la vez que distorsionados frente a la mirada ajena $-{ }^{37}$. No hay espacio aquí para tratar de realizar una descripción completa, ni siquiera general, del tipo de poder que está surgiendo de una era que, por otra parte, todavía está naciendo y cuyos contornos son aún difíciles de delimitar. El objetivo es más modesto, y tiene que ver con relacionar el régimen del saber que surge de la utilización del big data con determinadas prácticas de poder cuyas características recuerdan, aun sin coincidir del todo, a las del poder que Foucault describió en sus lecciones como pastoral.

\subsection{El poder pastoral en Foucault}

En las lecciones de 1978 recogidas bajo el título de Seguridad, Territorio, Población, Foucault dedica buena parte del texto a hablar del "poder pastoral", un tipo de gobierno de los hombres cuyo origen el autor sitúa en el Oriente Mediterráneo, y que, especialmente en su versión cristiana, va a servir de precedente para lo que en el Estado moderno se llamaría gubernamentalidad. Tipológicamente, un poder pastoral "se ejerce esencialmente sobre una multiplicidad en movimiento", una multiplicidad, por tanto, des-territorializada, que debe ser conducida de un lugar a otro por el pastor, el guía ${ }^{38}$. Por otra parte, se trata de un poder benévolo, "no tiene otra razón de ser que hacer el bien", no solo en el sentido de asegurar la subsistencia y el alimento, sino también en el sentido de que el pastor "cuida el rebaño" y de "los individuos del rebaño, vela por que las ovejas no sufran, va a buscar a las extraviadas, cura las heridas" 39 . Es un poder que "vela" por aquellos a los que guía: en el sentido "de la vigilancia del mal que puede hacerse, pero sobre todo de las desventuras que puedan sobrevenir", "vela porque las cosas sean lo mejor posible para cada uno de los integrantes del rebaño" ${ }^{\prime 40}$.

El poder pastoral es, además, un poder individualizador: "El pastor — prosigue Foucault - dirige todo el rebaño, pero solo puede hacerlo bien con la condición de que ni una sola de las ovejas se le escape. El pastor cuenta las ovejas, las cuenta por la mañana, en el momento de conducirlas a la pradera, las cuenta por la noche para saber si están todas, y se ocupa de ellas una por una". Omnes et singulatim: "El pastor debe tener los ojos puestos sobre todos y cada uno", debe salvarlos a todos del infortunio, con la única excepción de que cuando el rebaño está en peligro por una de las ovejas, la oveja negra, entonces esta puede ser sacrificada, excluida o abandonada

37 Harcourt, Exposed, pp. 107 y ss.; Javier Cigüela, "Poder y Subjetividad en la era digital (Reseña de Bernard E. Harcourt, Exposed. Desire and Disobedience in the Digital Age, Harvard University Press, 2016)": Isegoria, n. 55 (2016), pp. 735-739.

38 Michel Foucault, Seguridad, Territorio, Población, Fondo de Cultura Económica, Buenos Aires, 2006, pp. 154 y ss.

39 Ibid., p. 156.

40 Ibidem. 
en beneficio del rebaño ${ }^{41}$. Por ello, una de las grandes discusiones del poder pastoral es la cuestión de cuándo una oveja se convierte en insalvable, cuándo puede contaminar al rebaño y cuándo se convierte, por tanto, en sacrificable. Además de ello, el poder pastoral tiene lo que Foucault llama una "responsabilidad analítica" por su rebaño: "Deberá rendir cuentas de todos los actos de cada una de sus ovejas, todo lo que pudo ocurrirle a cada una de ellas, todo el bien y el mal que estas pudieron hacer en cada momento", debe gestionar los méritos y los deméritos como si fueran suyos propios, y en ello va su propia salvación ${ }^{42}$.

El poder pastoral supone, por último, una determinada relación con la verdad, más bien, con la producción de verdad. Para el pastor:

No se trata solo de enseñar lo que corresponde saber y hacer. Y no se trata de enseñarlo a través de principios generales, sino por una modulación cotidiana...es preciso que la enseñanza pase por una observación, una vigilancia y una dirección ejercida en cada instante y de la manera menos discontinua posible sobre la conducta integral, total de las ovejas ${ }^{43}$.

Es más:

La vida cotidiana no debe ser el mero resultado de una enseñanza general y ni siquiera de un ejemplo. Es necesario encargarse efectivamente de ella y observarla, de modo que el pastor, a partir de esta vida cotidiana de sus fieles vigilada por él, debe forjar un saber perpetuo que será el saber del comportamiento de la gente y su conducción ${ }^{44}$.

Citando a San Pablo (circa 5-58 e. c.), Foucault expresa la amplitud de la mirada del Pastor: "Por más éxtasis que alcance en la contemplación de lo invisible, [el Pastor] hace descender su espíritu al lecho conyugal. Enseña a los esposos la conducta que tienen el deber de respetar en sus relaciones íntimas"45.

La verdad entronca aquí con la dirección de las conciencias, una dirección que no es episódica o voluntaria, como lo fue en el mundo antiguo, sino que es permanente y obligatoria. Además, sostiene Foucault, la función política del examen de conciencia - y sobre esto volverá en otro lugar al analizar el papel de la confesión ${ }^{46}$ — no es tanto la de favorecer el auto-dominio o la auto-curación sino la de volver a la oveja dependiente del pastor por la vía de confesar sus vulnerabilidades:

Uno sólo examina su conciencia para poder decir al director lo que ha hecho, lo que es, lo que ha sentido, las tentaciones a las cuales ha estado sometido, los malos pensamientos que ha abrigado, el examen de conciencia se hace entonces para marcar, anclar aún mejor la relación de dependencia con el otro ${ }^{47}$.

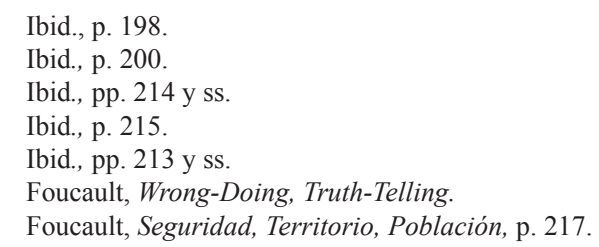


El régimen de verdad que ello constituye es muy específico: en su virtud "uno forjará a cada instante un discurso de verdad sobre sí mismo", "extraerá de sí mismo y producirá cierta verdad, y este será el elemento que le una a su director de conciencia"48.

Un tipo de poder, por tanto, a la vez colectivo e individualizador, que define "a cada instante el equilibrio, el juego y la circulación de los méritos y los deméritos"49, en una identificación analítica relacionada con la verdad confesada por el propio sujeto y no con su estatus. Un tipo de poder, en fin, que despojado de la retórica de la salvación y de la obediencia absoluta recuerda muy intensamente al poder tal y como está mutando en la era digital. A continuación explicaré en qué sentido.

\subsection{El Omnes et singulatim digital}

El primer aspecto en el que el poder en la era digital se asemeja a un poder pastoral tiene que ver con que también aquel se ejerce sobre una multiplicidad en movimiento y no sobre una población determinada y estática. Se pueden oponer las técnicas pastorales aquí a las disciplinarias. El disciplinario es un poder profundamente "isotópico" 50 , en él "cada elemento tiene su lugar determinado; tiene sus elementos subordinados y sus elementos superordinados" ${ }^{\prime 51}$. La escuela está distribuida por edades y capacidades, el ejército se distribuye por grados, la policía por rangos, igual que los presos o los trabajadores, a cada cual le corresponde un lugar que es a su vez expresión de una clasificación y jerarquización del conjunto, de un estatus. "A cada individuo su lugar, y en cada emplazamiento un individuo", "tantas parcelas como cuerpos o elementos a repartir", y "evitar las distribuciones por grupos": se acabaron los motines de presos y los corrillos de trabajadores perezosos, un espacio individualmente diferenciado que los divide a todos y los "sujeta" a su labor, sostiene Foucault ${ }^{52}$.

El poder pastoral es radicalmente diverso. En la medida en que se aplica a una multiplicidad en movimiento no se puede ejercer por emplazamiento y de forma estática, sino que ha de ejercerse por desplazamiento y conducción, ha de ser un poder dinámico que mueva al rebaño a determinados lugares y a determinadas prácticas a la vez que los saca de otros. Siendo así, resulta que las condiciones de nuestro mundo globalizado y de nuestra economía post-industrial e inmaterial precisamente lo que demandan son técnicas de control pastoral, des-territorializadas, que "presionen hacia una mayor apertura" de tiempo y espacio ${ }^{53}$. Las técnicas de control ya no se pueden aplicar al "topo disciplinario", recluido en celdas y demás espacios cerrados. En la actualidad el control se torna conducción, y según la conocida expresión de Gilles Deleuze (1925-1995), se ha de adaptar a sujetos que se comportan como serpientes, que se deslizan de un lugar a otro con su tecnología móvil y cuyo estado permanente es el movimiento ${ }^{54}$. La celda, el aula escolar, la casa, la habitación hospitalaria y demás lugares donde el poder se realizaba en

\footnotetext{
Ibidem.

Ibid., p. 218.

Michel Foucault, Vigilar y Castigar. Nacimiento de la prisión, Siglo XXI, Madrid, 2009, pp. 146 y ss.

Ibidem.

Ibidem.

Han, Psicopolítica, p. 32.

54 Gilles Deleuze, "Post-scriptum a las sociedades de control", en Conversaciones, Pre-textos, Valencia, 1999, pp. 277 y ss.; Han, Psicopolitica, pp. 31 y ss.
} 
y gracias $a$ una determinada distribución del espacio (ventanas en alto para que los escolares no se despisten, cristales reflectantes para que el preso no vea al carcelero, etc.) son complementadas, incluso progresivamente reemplazadas, por las pantallas digitales como lugares de normalización. El poder pastoral de la era digital ya no se transforma en espacio sino en dispositivo móvil. A medida que los espacios se vacían de signos, los dispositivos se llenan de alertas, y mientras nuestros cuerpos quedan liberados en superficies diáfanas es nuestra atención la que permanece capturada en la pantalla digital.

También la conducción propia del Pastor es replicada por el poder digital, que no solo registra nuestros movimientos, sino que los produce y los modifica. Por ejemplo, las Apps de mapas nos sugieren rutas más rápidas, más seguras y convenientemente patrocinadas pero si se equivocan nos pueden llevar incluso a la muerte, como le sucedió al turista italiano al que su GPS le llevó a una favela en Brasil donde fue asesinado en diciembre de $2016^{55}$. Por otra parte, tanto las técnicas de vigilancia política masiva desarrollada por Estados Unidos y otros muchos países como la recogida masiva de datos de la industria tecnológica constituyen técnicas de pastoreo digital. Se aplican a una multiplicidad de usuarios que van y vienen, que transitan de un lugar a otro proporcionando a cada paso información sobre su ubicación, sus planes y sus recorridos, pero se aplican a la vez sobre cada uno de ellos individualmente, generándose identidades virtuales (digital selfs) que van a ser analizadas en toda su particularidad ${ }^{56}$. Las apps que miden y analizan el rendimiento de los trabajadores, como la española Workmeter, cumplen igualmente el patrón: proporcionan un conocimiento exhaustivo sobre los flujos generales del grupo (productividad, empleo del tiempo, patrones de comportamiento), a la vez que lo hacen de cada uno de los trabajadores individuales (rendimiento y productividad comparativa, geo-localización personal, etc. $)^{57}$.

El poder pastoral digital también es un poder que cuida y que vela por su rebaño de usuarios, un poder benévolo. Si uno hace un somero análisis de los informes donde la recogida y el uso del big data son legitimados como tecnologías de saber-poder, reparará inmediatamente en que su omnisciencia y ubicuidad queda justificada por los cuidados que aporta. El poder digital, gracias a su conocimiento exhaustivo de nosotros y de las personas como nosotros con las que nos correlaciona y compara, es capaz, igual que se exige del pastor, de detectar cualquier amenaza y adelantarse a ella incluso antes de que se manifieste. Según un informe reciente, "el Big data es una herramienta importante a la hora de desarrollar nuevos tipos de cuidado (care) personalizado. Compañías como 23andme y Navigenics pueden proveer un análisis del genoma de la persona altamente detallado", información que puede ser útil para "customizar determinados tipos de tratamiento médico"58. De modo similar, la Georgia State University ha desarrollado un sistema llamado Graduation and Progression Success (GPS), mediante el cual realiza un seguimiento continuo de treinta y dos mil estudiantes. De cara a asegurar que concluyen satisfactoriamente el grado, un sistema de alertas detecta irre-

55 Ver al respecto: "Brasil: el terrible asesinato de un turista italiano en una peligrosa favela de Río de Janeiro a la que llegó llevado por su GPS": $B B C$ (9-12-2016). Disponible en: http://www.bbc.com/mundo/noticias-americalatina-38270594 (31-5-2017).

56 Harcourt, Exposed, p. 137.

57 Ver aquí los servicios que ofrece: http://web.workmeter.com/es/index.html.

58 "Finally, data is an important tool in developing new types of personalized health care. Companies like 23andme and Navigenics can provide highly detailed genetic analysis of a persons's genome - information that may be helpful in customizing certain types of medical care”. Bollier, The Promise and Peril of Big Data, p. 27. 
gularidades que el sistema correlaciona con el fracaso escolar (malas planificaciones o calificaciones, absentismo, etc.). Gracias a ello se activa el protocolo de actuación con suficiente antelación como para evitar que el problema vaya a mayores. El sistema se aplica con especial intensidad sobre las minorías negras y latinas, sobre las que el seguimiento es mayor y, según el informe, especialmente exitoso ${ }^{59}$.

Igual que sucede con el poder pastoral, el poder digital, igual de ubicuo y omnipresente, tan continuo y predictivo como aquel, es por encima de todo un poder benévolo y beneficioso: incluso las pequeñas molestias que pueda ocasionar, en términos de falta de autonomía o de exceso de paternalismo, quedan en nada si se tiene en cuenta la magnitud de los cuidados que aporta. Las pretensiones de privacidad o de autodeterminación que un día fundamentaron nuestra vida social, hoy son avasalladas por una expectativa de ser cuidados y de ser exitosos en nuestras empresas. Es cierto que el sistema que surge de dicho saber-poder se parece mucho a un Gran hermano orwelliano, pero a diferencia de este el Gran hermano digital "tiene un aspecto amable": "La eficacia de su vigilancia — sostiene Han — reside en su amabilidad"60, precisamente porque es ella la que legitima su carácter invasivo y constante.

El cuidado recae sobre la multiplicidad - millones en el caso del Health care 2.0., treinta y dos mil en el caso universitario-, pero también es individualizado y personalizado. Es más, como el buen pastor, el poder digital vela especialmente por el débil, por el que se puede descarrilar más fácilmente. Ahora bien, el carácter generalista del poder pastoral digital no excluye el sacrificio de la oveja irrecuperable, si así lo exige la supervivencia del conjunto. Así, el destino que han corrido personas como Edward Snowden, Julian Assange o Chealsea Manning, incluso Aaron Swartz, todos ellos excluidos o expulsados precisamente por tratar de revelar los arcanos del poder al público afectado, indica que también la era digital produce sus expulsados y sus excluidos. El panóptico se convierte eventualmente, según la expresión de Didier Bigo ${ }^{61}$, en un banóptico (ban en inglés significa "expulsión", "proscripción"): el mismo poder que cuida benévola y continuamente del rebaño separa a quienes pueden contaminarlo. La explicación de tal separación no será la ira del poder o la violencia temible propia de los reyes y los dioses soberanos. Según Foucault, todo ello se borra cuando se trata de un poder pastoral ${ }^{62}$. Aquí estamos ante una mera cuestión de seguridad y mantenimiento del conjunto, de nuevo una cuestión, por tanto, de benevolencia, la que obliga a abandonar a quienes pueden poner en riesgo a la colectividad.

Ahora bien, para que todo lo anterior se realice exitosamente es menester producir un determinado régimen de verdad, tanto más fuerte como más difícil es controlar a una multiplicidad en movimiento. Dicho de otro modo, si la producción de un conocimiento sobre los sujetos era más o menos importante cuando se trataba de individuos con trabajos, casas y conductas estables, lo cierto es que se va a convertir

59 Martin Kurzweil y Derek Wu, "Building a Pathway to Student Success at Georgia State University": Ithaka S\&R Case Study (2015). Disponible en: http://www.sr.ithaka.org/publications/building-a-pathway-to-studentsuccess-at-georgia-state-university/ (18-9-2017); Jon Marcus, "Colleges Use Data to Predict Grades and Graduation": The Hechinger Report (10-12-2014). Disponible en: http://hechingerreport.org/like-retailers-tracking-trends-colleges-use-data-predict-grades-graduations/ (18-9-2017).

60 Han, Psicopolitica, p. 61.

${ }_{61}$ Didier Bigo, "Globalized (in)Security: the Field and the Ban-opticon" (2006). Disponible en: http://www. people.fas.harvard.edu/ ces/conferences/muslims/Bigo.pdf (11-1-2017).

62 Foucault, Seguridad, Territorio, Población, p. 157. 
en crucial cuando se trata de individuos líquidos que fluyen de un lugar a otro y que carecen de estabilidad, tal y como sucede en la actualidad ${ }^{63}$. El poder digital, en la línea del pastoral, va a ser de carácter profundamente analítico, tanto a nivel macro como a nivel micro.

En lo relativo al nivel macro, el principal objetivo del régimen de verdad digital es traducir en datos cuantificables y utilizables los flujos masivos, informes, cambiantes y dinámicos de personas (migrantes, trabajadores, turistas, conductores, consumidores, etc.), de bienes (dinero y otros activos financieros, mercancías, conocimientos, alimentos, etc.), como también de peligros (enfermedades, accidentes, riesgos medioambientales, drogas, amenazas terroristas, etc.). No solo en el sentido ex post facto, como recogida de flujos existentes o pasados, sino también ex ante, en el sentido de que se ha de tratar de configurar una expectativa de futuro respecto a dichos flujos. Como se ha dicho ya, buena parte del carácter mitológico del big data como saber-poder reside en su capacidad para "leer el futuro", anticiparse y prever peligros.

Una de las razones por las que el régimen de poder-saber producido por la era digital es diverso a los anteriores es que su capacidad de captar lo macro no mitiga su capacidad de profundización. Según explica Manovich ${ }^{64}$, la metodología propia de la era estadística tenía que elegir entre el "surface data", extraído de una gran masa de población, y el "deep data", extraído de unos pocos individuos. Cuanto mayor era el rango de población analizado menor era la profundidad del conocimiento extraído, y viceversa. La utilización de muestras potencialmente extrapolables, lo que constituía el principal remedio para dicha limitación, no producía más que una "foto pixelada" de la realidad: una representación incompleta de algo respecto de lo que no se tiene un conocimiento real. La concurrencia actual de los datos masivos con las "supercomputadoras" y los algoritmos permite saltar por encima de esa limitación. Hoy ya es posible extraer "deep data" de millones y millones de personas, y aunque el conocimiento no sea tan profundo como el que podría corresponder a un etnógrafo en el terreno, para determinadas utilidades sociales y políticas lo que el big data ofrece es más que suficiente, como también lo es para proveer la referida ilusión de control.

En ese sentido, y todavía dentro del nivel macro, el pastor digital no solo da cuenta de sus ovejas, su estado y sus movimientos al principio y al final de cada jornada, sino que lo hace a cada instante y sin discontinuidades. Es posible que nosotros solo nos demos cuenta de dicho control de tanto en tanto (como cuando Facebook o Spotify nos ofrecen un resumen de nuestra actividad al final de cada año, o cuando Google nos avisa de una cita inminente en el calendario), pero lo cierto es que el análisis produce un mapa de datos diferente a cada instante. Dicho con un ejemplo: el trabajador de Amazon cuyo rendimiento es monitorizado y comparado con el resto no recibe un informe al final del día o de la semana, sino que esa información se actualiza a cada instante y, según se reveló en una investigación reciente ${ }^{65}$, se le muestra al propio trabajador de modo permanente en su pantalla mientras empaqueta. Gracias a ello puede ver si su línea de rendimiento está por debajo de la media actual de trabajadores o si está por encima de ella, como también puede recibir avisos e informaciones instantáneas de sus superiores.

63 Zigmunt Bauman y David Lyon, Vigilancia líquida, Paidós, Barcelona, 2013.

${ }_{64}$ Manovich, "Trending: The Promises and the Challenges of Big Social Data", pp. 3 y ss.

65 Sarah O'Connor, “Amazon Unpacked": Financial Times Magazine (8-2-2013). Disponible en: https:// www.ft.com/content/ed6a985c-70bd-11e2-85d0-00144feab49a (11-1-2017). Sobre ello: Harcourt, Exposed, p. 195. 
El saber que se genera en la era digital es analítico, efectivamente, pero además es más instantáneo, continuo y previsor que el que se pudo manifestar en la era estadística y con anterioridad. Ahora bien, entrando ya al nivel micro, resulta que el poder pastoral digital es capaz también de mayor profundidad y subjetivación, precisamente porque el régimen de verdad que produce está intensamente individualizado. Las técnicas actuales de análisis de los usuarios - tengan estos el papel de consumidores, votantes o ciudadanos - incorporan lo que se ha venido a llamar el microtargeting, es decir, la capacidad de quien gestiona la base de datos masivos de acumular tantos conocimientos sobre los almacenados que la comunicación con ellos no ha de ser estandarizada (como en la era estadística) sino que puede llegar a ser personalizada. La conocida empresa de datos Acxiom presume, por ejemplo, de tener un "conocimiento $360^{\circ "}$ de los usuarios cuyos datos posee; más de quinientos millones de consumidores, según sus directivos, con más de mil quinientos datos de cada uno de ellos ${ }^{66 / 67}$. Gracias a ese saber acumulado, quien quiera comprar dichos datos - empresas, gobiernos, centros de inteligencia, partidos políticos, etc.- tendrá la posibilidad de realizar una comunicación estratégica altamente sofisticada con cada uno de los usuarios, anticipándose en gran medida a su expectativa. Estos, como no pueden hacer de otro modo dado el carácter profundamente asimétrico de la relación, seguramente hayan consentido todo el proceso de antemano, al visitar la página web y aceptar las cookies, o al dar el consentimiento a la transferencia de información al descargar la aplicación en cuestión.

Según Han, todo lo anterior hace del régimen del saber actual una "microfísica del poder", en la que los poderes políticos o económicos saben tanto de los ciudadanos que son capaces de amoldar su "oferta" a su subjetividad ${ }^{68}$. Produce, además, una "psicopolítica movida por datos", en el sentido de que no solo se amolda a nuestra subjetividad, sino que la produce conduciéndola - mediante recomendaciones personalizadas - hacia lugares, experiencias o gustos económicos o políticamente explotables. Como es propio del poder pastoral, es el régimen de la verdad confesada lo que vincula al poder con el súbdito, una verdad que no es extraída disciplinariamente, sino más bien ofrecida gratuitamente por parte de la "oveja digital" para favorecer una mejor conducción por parte del pastor. Poco importa que lo que en la era pastoral se llamase "salvación" se denomine hoy "experiencia de usuario", o que el término "confesión" se substituya por "comunicación", pues el proceso de subjetivación e interiorización del poder en la psique del afectado es tanto o más fuerte como lo fue entonces.

\section{Conclusión}

El big data se ha integrado hasta tal punto en nuestro medio-ambiente social que sus efectos comienzan a ser particularmente intensos tanto en la esfera personal como en

66 Natasha Singer, "Mapping, and Sharing, the Consumer Genome": The New York Times (16-6-2012). Disponible en: http://www.nytimes.com/2012/06/17/technology/acxiom-the-quiet-giant-of-consumer-database-marketing. html?_r=0 (11-1-2017).

67 Sobre el caso de Acxiom: Han, Psicopolitica, pp. 86 y ss., quien argumenta acertadamente que no es que este tipo de acumulación de datos genere - como creen los dataístas - "mejor" o "mayor" conocimiento, sino más bien conocimiento de otro tipo: no narrativo, aperspectivista, y en cierto modo cualitativamente pobre; Harcourt, Exposed, pp. 199 y ss.

68 Han, Psicopolítica, pp. 95 y ss. 
la social y política. Sus efectos positivos y beneficiosos, en términos de mejora de las comunicaciones y de la capacidad analítica, están ampliamente acreditados y no son objeto de discusión en el presente artículo. Ha interesado aquí abordar más bien el modo en que un saber constituido por datos masivos, aparentemente neutros y funcionales, estalla en múltiples direcciones y adquiere un carácter mitológico. Estalla, en primer lugar, en la fundación de un saber que va a ser políticamente validado y que va a condicionar las decisiones políticas: quien controle los datos y los algoritmos que los regulan va a disponer de una presunción de autoridad, y precisamente por ello, convertirá dicho saber en el arcano o el secreto del poder, clausurado bajo amenaza de exclusión. Poco importa que los datos incidan realmente en una mejor decisión (muy posiblemente lo hará), pues lo relevante desde el punto de vista político y discursivo es la "ilusión de control" que generan, el modo en que proporcionan seguridad al decisor y al afectado en contextos de alta incertidumbre y complejidad. Estalla, en segundo lugar, en la fundación de un nuevo tipo de poder, aquí llamado pastoral digital, que va a conducir a las masas móviles a lugares donde el movimiento mismo pueda ser explotado económica o políticamente. Para ello se va a servir de la tecnología móvil que el propio usuario lleva incorporada al cuerpo, cuyas pantallas van a servir como medio para capturar su atención y para hacer circular verdades — también rentables - sobre sí mismo y sobre los demás (ubicación, gustos, recorridos, consumo, estados de ánimo, estilo de vida y todo lo imaginable). El modo inconsciente mediante el cual el usuario transmite verdades sobre sí mismo hace que esta tecnología sea mucho más eficaz que las anteriores, particularmente que la estadística. En la medida en que el usuario no se siente interrogado sino que más bien se ve como partícipe de una comunicación libre, baja la guardia y queda por ello más expuesto frente a la mirada analítica de los diferentes poderes.

La política de la era digital replica, en cierto sentido, el tipo de dominación por conducción propia del poder pastoral: camuflándose en un régimen de verdad aparentemente neutro - el de los datos - y en una tecnología digital que se le aparece al usuario como el vehículo a experiencias positivas —iPhone, iPad, tablets, etc. - , los poderes consiguen ser ubicuos a la vez que imperceptibles, justamente lo que se necesita para estabilizar a las masas móviles que conforman el rebaño global contemporáneo.

\section{Referencias bibliográficas}

Anderson, Chris, "The End of Theory: The Data Deluge Makes the Scientific Method Obsolete": Wired (23-6-2008). Disponible en: https://www.wired.com/2008/06/pbtheory/ (11-1-2017).

Bantjes, Marian, “The Petabyte Age: Because More Isn't Just More-More Is Different”: Wired (23-6-2008). Disponible en: https:/www.wired.com/2008/06/pb-intro/ (11-1-2017).

Bauman, Zigmunt y Lyon, David, Vigilancia líquida, Paidós, Barcelona, 2013.

Bigo, Didier, "Globalized (in)Security: the Field and the Ban-opticon (2006). Disponible en: http://www.people.fas.harvard.edu/ ces/conferences/muslims/Bigo.pdf (11-1-2017).

Bollier, David (Rapporteur), The Promise and Peril of Big Data, Aspen Institute, Washington, 2010.

Boyd, Danah y Crawford, Kate, "Critical Questions for Big Data": Information, Communication \& Society, vol. 15, n. 5 (2012), pp. 662-679. 
Braidotti, Rosi, Lo Posthumano, Gedisa, Barcelona, 2015.

Bratton, Benjamin, The Stack. On Software and Sovereignty, MIT Press, Cambridge, London, 2015.

Carr, Nicholas, Superficiales. ¿Qué está haciendo internet con nuestras mentes?, Taurus, Madrid, 2011.

Cigüela, Javier, "Poder y Subjetividad en la era digital (Reseña de Bernard E. Harcourt, Exposed. Desire and Disobedience in the Digital Age, Harvard University Press, 2016)": Isegoria, n. 55 (2016), pp. 735-739.

Deleuze, Gilles, "Post-scriptum a las sociedades de control", en Conversaciones, Pre-textos, Valencia, 1999.

Derrida, Jaques, Fuerza de Ley. El "fundamento místico de la autoridad", Tecnos, Madrid, 2010.

Han, Byung-Chul, La sociedad de la transparencia, Herder, Barcelona, 2013.

- Psicopolítica, Herder, Barcelona, 2014.

Harari, Nuval Yoah, Homo Deus. Breve historia del mañana, Debate, Barcelona, 2016.

Harcourt, Bernard E., Exposed. Desire and Disobedience in the Digital Age, Harvard University Press, Cambridge, 2016.

Foucault, Michel, Power/Knowledge. Selected Interviews \& Other Writings, 1972-1977, The Harvester Press/Vintage Books, New York, 1980.

- Los anormales, Akal, Madrid, 2001.

- Seguridad, Territorio, Población, Fondo de Cultura Económica, Buenos Aires, 2006.

- La verdad y las formas jurídicas, Gedisa, Barcelona, 2008.

- Vigilar y Castigar. Nacimiento de la prisión, Siglo XXI, Madrid, 2009.

- Wrong-Doing, Truth-Telling. The function of avowal in Justice, University of Chicago Press, Chicago, London, 2014.

Kurzweil, Martin y Wu, Derek, "Building a Pathway to Student Success at Georgia State University", Ithaka S\&R Case Study (23-4-2015). Disponible en: http://www.sr.ithaka.org/ publications/building-a-pathway-to-student-success-at-georgia-state-university/ (18-9-2017).

Leibniz, Gottfried W., Leibniz: Selections, ed. y trad. de P. P. Wiener, Scribner, New York, 1951.

Luhmann, Niklas, Das Recht der Gesellschaft, Suhrkamp, Frankfurt, 1995.

— Organización y decisión, Herder/Universidad Iberoamericana, México, D.F., 2010.

Manovich, Lev, "Trending: The Promises and the Challenges of Big Social Data" (2011). Disponible en: http://manovich.net/content/04-projects/067-trending-the-promises-andthe-challenges-of-big-social-data/64-article-2011.pdf (11-1-2017).

- El software toma el mando, UOC Press, Barcelona, 2013.

Mantelero, Alessandro, "Smart cities, movilidad inteligente y protección de los datos personales": IDP. Revista de Internet, Derecho y Política, n. ${ }^{\circ} 21$ (2015), pp. 37-49.

Marcus, Jon, "Colleges Use Data to Predict Grades and Graduation", The Hechinger Report (10-12-2014). Disponible en: http://hechingerreport.org/like-retailers-tracking-trendscolleges-use-data-predict-grades-graduations/ (18-9-2017).

McLuhan, Marshall, Comprender los medios de comunicación. Las extensiones del ser humano, Paidós, Barcelona, 1996.

— "La entrevista de Playboy", en Carlos Scolari (ed.), Ecología de los medios. Entornos, evoluciones e interpretaciones, Gedisa, Barcelona, 2015, pp. 45-96.

O’Connor, Sarah, “Amazon Unpacked”: Financial Times Magazine (8-2-2013). Disponible en: https://www.ft.com/content/ed6a985c-70bd-11e2-85d0-00144feab49a (11-1-2017).

Pasquale, Frank, The Black Box Society. The Secret Algorithms that Control Money and Information, Harvard University Press, Cambridge, 2015. 
Shmueli, Galit, “To Explain or to Predict?”: Statistical Science, vol. 25, n. o 3 (2010), pp. 289310.

Singer, Natasha, "Mapping, and Sharing, the Consumer Genome": The New York Times (166-2012). 2012. Disponible en: http://www.nytimes.com/2012/06/17/technology/acxiomthe-quiet-giant-of-consumer-database-marketing.html?_r=0 (11-1- 2017).

Willke, Helmut, Smart Governance: Governing the Glob̆al Knowledge Society, Campus Verlag, New York, 2007. 